H. MAEDA

KODAI MATH. J.

18 (1995), $187-197$

\title{
NEF LINE BUNDLES ON ALGEBRAIC SURFACES
}

\author{
HIDETOSHI MAEDA
}

\section{$\S 0$. Introduction}

In this paper we assume throughout that the ground field $k$ is algebraically closed and of characteristic $p \geqq 0$.

Let $X$ be a projective surface and $L$ a line bundle on $X$. When $L$ is nef (i. e., $L C \geqq 0$ for all integral curves $C$ on $X)$, the pair $(X, L)$ will be called a semipolarized surface. We say that two semipolarized surfaces $\left(X_{1}, L_{1}\right)$ and $\left(X_{2}, L_{2}\right)$ are birationally equivalent if there is a projective surface $W$ with birational morphisms $f_{2}: W \rightarrow X_{2}(i=1,2)$ such that $f_{1}^{*} L_{1}=f_{2}^{*} L_{2}$. Moreover, if $X$ is normal, the sectional genus $g(X, L)$ of the semipolarized normal surface $(X, L)$ is given by the formula $2 g(X, L)-2=\left(\boldsymbol{\omega}_{X}+L\right) L$, where $\omega_{X}$ is the canonical sheaf of $X$.

Lanteri and Palleschi proved the following on the assumption that $L$ is an ample line bundle on a smooth complex projective surface $X$.

THEOREM 0.1 ([LP], Remark 1.3). Let $L$ be an ample line bundle on a smooth complex projective surface $X$. Then one of the following holds.

(1) $K_{X}+L$ is nef for the canonical bundle $K_{X}$ of $X$.

(2) $(X, L) \cong\left(\boldsymbol{P}^{2}, \mathcal{O}_{\boldsymbol{P}}(1)\right)$.

(3) $(X, L) \cong\left(\boldsymbol{P}^{2}, \mathcal{O}_{\boldsymbol{P}}(2)\right)$.

(4) $(X, L)$ is a scroll over a smooth curve (For the definition of a scroll, see $\S 1$.).

THEOREM 0.2 ([LP], Remark 1.1 and Corollary 2.3). Let $L$ be an ample line bundle on a smooth complex projective surface $X$. Then $g(X, L) \geqq 0$. Moreover, if $g(X, L)=0$, then $(X, L)$ is one of the following.

(1) $(X, L) \cong\left(\boldsymbol{P}^{2}, \mathcal{O}_{\boldsymbol{P}}(1)\right)$.

(2) $(X, L) \cong\left(\boldsymbol{P}^{2}, \mathcal{O}_{\boldsymbol{P}}(2)\right)$.

(3) $(X, L)$ is a scroll over $\boldsymbol{P}^{1}$.

THEOREM 0.3 ([LP], Corollary 2.4). Let $L$ be an ample line bundle on a smooth complex projective surface $X$. If $g(X, L)=1$, then $(X, L)$ is one of the following.

Received May 19, 1994 ; revised June 20, 1994. 
(1) $(X, L)$ is a scroll over an elliptic curve.

(2) $X$ is a Del Pezzo surface and $L=-K_{X}$.

It seems very natural to carry out similar research in the case when $L$ is simply supposed to be a nef line bundle because the nefness is the degenerate condition of ampleness. The purpose of this paper is to generalize these results to semipolarized surfaces over an algebraically closed field of arbitrary characteristic. The precise statements of our results are as follows:

THEOREM 1. Let $(X, L)$ be a semipolarized surface. Then there exist a smooth projective surface $Y$ and a nef line bundle $M$ on $Y$ such that the semipolarized surface $(Y, M)$ is birationally equivalent to $(X, L)$ and satisfies one of the following.

(1) $K_{Y}+M$ is nef.

(2) $(Y, M) \cong\left(\boldsymbol{P}^{2}, \mathcal{O}_{\boldsymbol{P}}\right)$.

(3) $(Y, M) \cong\left(\boldsymbol{P}^{2}, \mathcal{O}_{\boldsymbol{P}}(1)\right)$.

(4) $(Y, M) \cong\left(\boldsymbol{P}^{2}, \mathcal{O}_{\boldsymbol{P}}(2)\right)$.

(5) $(Y, M)$ is a scroll over a smooth curve.

(6) $Y$ is a $\boldsymbol{P}^{1}$-bundle over a smooth curve $C$ and $M$ is the pull-back of $a$ line bundle of non-negative degree on $C$.

THEOREM 2. Let $(X, L)$ be a semipolarized normal surface. Then $g(X, L)$ $\geqq 0$ unless $Y$ is a $\boldsymbol{P}^{1}$-bundle over a smooth curve $C$ and $M \cong \pi^{*} B$ for some line bundle $B$ on $C$ with $\operatorname{deg} B \geqq 2$, where $(Y, M)$ is as in the Theorem 1 and $\pi$ is the projection $Y \rightarrow C$. Moreover, if $g(X, L)=0$, then the pair $(Y, M)$ as in the Theorem 1 is one of the following.

(1) $(Y, M) \cong\left(\boldsymbol{P}^{2}, \boldsymbol{O}_{\boldsymbol{P}}(1)\right)$.

(2) $(Y, M) \cong\left(\boldsymbol{P}^{2}, \mathcal{O}_{\boldsymbol{P}}(2)\right)$.

(3) $(Y, M)$ is a scroll over $\boldsymbol{P}^{1}$.

(4) $Y$ is a $\boldsymbol{P}^{1}$-bundle over a smooth curve $C$ and $M \cong \pi * B$ for some line bundle $B$ on $C$ with $\operatorname{deg} B=1$.

THEOREM 3. Let $(X, L)$ be a semipolarized normal surface with $g(X, L)=1$. Then the pair $(Y, M)$ as in the Theorem 1 is one of the following unless (0) $M$ is numerically trivial.

(1) $Y$ is a minimal surface with Kodaira dimension 1 and $K_{Y} M=M^{2}=0$.

(2) $K_{Y}$ is numerically trivial and $M^{2}=0$.

(3) There is an indecomposable vector bundle $\mathcal{E}$ on an elliptıc curve $C$ with $c_{1}(\mathcal{E})=1$ such that $Y \cong \boldsymbol{P}_{C}(\mathcal{E}) . \quad M=a H+\pi^{*} B$ for some line bundle $B$ of degree $-a / 2$ on $C$ and $a \geqq 2$, where $H$ is the tautological line bundle on $Y$ and $\pi$ is the projection $Y \rightarrow C$.

(4) There is a vector bundle $\mathcal{E}$ on an elliptic curve $C$ with $c_{1}(\mathcal{E})=0$ such that $Y \cong \boldsymbol{P}_{C}(\mathcal{E}) . \quad M=a H+\pi^{*} B$ for some line bundle $B$ of degree 0 on $C$ and $a \geqq 2$, where $H$ and $\pi$ are as in (3). 
(5) $Y$ is a weak Del Pezzo surface and $M=-K_{Y}$.

(6) $Y$ is the blow-up of $Z$ at a point, where $Z$ is a weak Del Pezzo surface with $\left(-K_{Z}\right)^{2}=1 . \quad M=-a K_{Y}$ and $a \geqq 1$.

(7) $(Y, M)$ is a scroll over an elliptic curve.

This type of Theorems have been obtained by Fujita $[F]$ for the case in which $L$ is a nef and big line bundle on a projective variety of dimension $\leqq 3$ defined over an algebraically closed field of characteristic zero.

I would like to thank Professor Antonio Lanteri for his encouragement during the preparation of this paper. I would also like to express my gratitude to the referee for useful comments.

\section{$\S 1$. Preliminaries}

Let $X$ be a projective surface defined over an algebraically closed field $k$ of characteristic $p \geqq 0$. The group of line bundles on $X$ is denoted by Pic $(X)$. The tensor products of line bundles are denoted additively. Let $Z_{1}(X)$ be the free abelian group generated by integral curves on $X$. The intersection pairing gives a bilinear map $\operatorname{Pic}(X) \times Z_{1}(X) \rightarrow Z$ and the numerical equivalence $\equiv$ is defined so that the pairing $((\operatorname{Pic}(X) / \equiv) \otimes \boldsymbol{Q}) \times\left(\left(Z_{1}(X) / \equiv\right) \otimes \boldsymbol{Q}\right) \rightarrow \boldsymbol{Q}$ is non-degenerate. The closed cone of curves $\overline{N E}(X)$ is the closed convex cone generated by effective 1-cycles in the $\boldsymbol{R}$-vector space $\left(Z_{1}(X) / \equiv\right) \otimes \boldsymbol{R} . \quad L \in \mathrm{Pic}(X)$ is called nef if the numerical class of $L$ in $(\operatorname{Pic}(X) / \equiv) \otimes \boldsymbol{R}$ gives a non-negative function on $\overline{N E}(X)-\{0\}$. A nef line bundle $L$ is called big if $L^{2}>0$.

Let $X$ be a smooth projective surface. The canonical bundle of $X$ is denoted by $K_{X}$. Let $Z$ be a 1-cycle on $X$. We denote by $[Z]$ the numerical class of $Z$ in $\left(Z_{1}(X) / \equiv\right) \otimes \boldsymbol{R}$. A half line $R=\boldsymbol{R}_{+}[Z]\left(\boldsymbol{R}_{+}=\{x \in \boldsymbol{R} \mid x \geqq 0\}\right)$ in $\overline{N E}(X)$ is called an extremal ray if

(1) $K_{X} Z<0$, and

(2) if $z_{1}, z_{2} \in \overline{N E}(X)$ satisfy $z_{1}+z_{2} \in R$, then $z_{1}, z_{2} \in R$.

A rational curve $l$ on $X$ is called an extremal rational curve if $\left(-K_{X}\right) l \leqq 3$ and $\boldsymbol{R}_{+}[l]$ is an extremal ray. Let

$$
\overline{N E}(X)^{+}=\left\{z \in \overline{N E}(X) \mid K_{X} z \geqq 0\right\} .
$$

Then we have the following basic theorem.

THEOREM 1.1 (Cone Theorem). Let $X$ be a smooth projective surface. Then $\overline{N E}(X)$ is the smallest closed convex cone containing $\overline{N E}(X)^{+}$and all the extremal rays:

$$
\overline{N E}(X)=\overline{N E}(X)^{+}+\Sigma R_{\jmath},
$$

where the $R$, are extremal rays of $\overline{N E}(X)$ for $X$. Furthermore, every extremal ray is spanned by a numerical class of an extremal rational curve. 
For the proof, we refer to [Mo], Theorem 1.5. We need also the following theorem.

THEOREM 1.2. Let $X$ be a smooth projective surface. Then an irreducible curve $l$ on $X$ is an extremal rational curve if and only if

(1) $X \cong \boldsymbol{P}^{2}$ and $l$ is a straight line,

(2) $X$ is a $\boldsymbol{P}^{1}$-bundle over a smooth curve $C$ and $l$ is a fiber of $X \rightarrow C$, or

(3) l is a (-1)-curve.

For the proof, we refer to [Mo], Theorem 2.1.

(1.3) Let $Y$ be a $\boldsymbol{P}^{1}$-bundle over a smooth curve $C$ of genus $g$. Then there exists a vector bundle $\mathcal{E}$ of rank two on $C$ such that $Y \cong \boldsymbol{P}_{C}(\mathcal{E})$. We may assume that $h^{0}(\mathcal{E}) \neq 0$ and that $h^{0}(\mathcal{E} \otimes L)=0$ for all line bundles $L$ on $C$ with $\operatorname{deg} L<0$. In this case $\mathcal{E}$ is said to be normalized. Of course $\mathcal{E}$ is not necessarily determined uniquely, but the integer $e=-c_{1}(\mathcal{E})$ is an invariant of $Y$. If $\mathcal{E}$ is decomposable, then $\mathcal{E} \cong \mathcal{O}_{C} \oplus L$ for some line bundle $L$ on $C$ with deg $L \leqq 0$. Therefore $e \geqq 0$. All values of $e \geqq 0$ are possible. If $\mathcal{E}$ is indecomposable, then $-g \leqq e \leqq 2 g-2$. Let $H$ be the tautological line bundle on $Y$. Then there is a section $C_{0}$ such that $C_{0} \in|H|$. Let $\pi$ be the projection $Y \rightarrow C$ and $F$ a fiber of $\pi$. Then every line bundle $M$ on $Y$ is numerically equivalent to $a C_{0}+b F$ for some integers $a, b$. For details we refer to $[\mathrm{H}]$. A necessary and sufficient condition for $M$ to be nef is stated as follows.

LEMMA 1.4. Let $Y$ be a $\boldsymbol{P}^{1}$-bundle over a smooth curve $C$ with invariant $e \geqq 0$. Then a line bundle $M \equiv a C_{0}+b F$ is nef if and only if $a \geqq 0$ and $b \geqq a e$.

For the proof, we refer to $[\mathrm{Ma}]$, Lemma 1.3.

LEMMA 1.5. Let $Y$ be a $\boldsymbol{P}^{1}$-bundle over a smooth curve $C$ of genus $g$ with invariant $e<0$. Assume furthermore either char $k=0$ or $g \leqq 1$. Then a line bundle $M \equiv a C_{0}+b F$ is nef if and only if $a \geqq 0$ and $b \geqq(1 / 2) a e$.

For the proof, we refer to $[\mathrm{Ma}]$, Lemma 1.4 .

A semipolarized surface is a pair $(X, L)$ consisting of a projective surface $X$ and a nef line bundle $L$ on $X$. A semipolarized surface $(X, L)$ is said to be a scroll over a smooth curve $C$ if $X$ is a $P^{1}$-bundle over $C$ and $L F=1$ for any fiber $F$ of $X \rightarrow C$. A smooth projective surface $X$ is called weak Del Pezzo if $-K_{X}$ is nef and big; it is well-known (see for example [D]) that $X$ is then either $\boldsymbol{P}^{1} \times \boldsymbol{P}^{1}$ or $\Sigma_{2}$ or isomorphic to $\boldsymbol{P}^{2}$ blown up $\leqq 8$ times in weakly general position, where $\Sigma_{2}$ is the second Hirzebruch surface. 


\section{$\S 2$. Proof of the Theorem 1}

Let $g: Z \rightarrow X$ be a desingularization of $X$. Considering the pull-back $g * L$, we may assume from the start that $X$ is smooth because two semipolarized surfaces $(X, L)$ and $\left(Z, g^{*} L\right)$ are birationally equivalent.

If $K_{X}+L$ is nef, then we set $(Y, M)=(X, L)$.

Assume that $K_{X}+L$ is not nef. Then, since $K_{X}+L$ is not non-negative on $\overline{N E}(X)-\{0\}$, by the cone Theorem 1.1 we can find an extremal rational curve $l$ with $\left(K_{X}+L\right) l<0$, and so

$$
0 \leqq L l<\left(-K_{X}\right) l .
$$

From Theorem 1.2 there are three cases:

(1) $X \cong \boldsymbol{P}^{2}$ and $l$ is a straight line.

(2) $X$ is a $P^{1}$-bundle over a smooth curve $C$ and $l$ is a fiber $F$ of $\pi: X \rightarrow C$.

(3) $l$ is a $(-1)$-curve $E$.

In case $(1) L \cong \mathcal{O}_{\boldsymbol{P}}, \mathcal{O}_{\boldsymbol{P}}(1)$ or $\mathcal{O}_{\boldsymbol{P}}(2)$, since $\left(-K_{X}\right) l=3$. In case (2) we can write

$$
L=a H+\pi * B
$$

for some line bundle $B$ on $C$, where $H$ is the tautological line bundle on $X$. Since $\left(-K_{X}\right) F=2$, we have

$$
0 \leqq L F=a \leqq 1 .
$$

If $a=1$, then $(X, L)$ is a scroll over $C$; assume that $a=0$. Then $L=\pi * B$, so $\operatorname{deg} B \geqq 0$ because $L$ is nef. In every case we set $(Y, M)=(X, L)$.

Now we consider the case (3). The contraction criterion of Castelnuovo and Enriques says that $E$ can be contracted to a point of another smooth projective surface $X^{\prime}$ with the Picard number $\rho\left(X^{\prime}\right)=\rho(X)-1$; let $f: X \rightarrow X^{\prime}$ be the blow-down of $E$. Since $\left(-K_{X}\right) E=1$, we have $L E=0$; thus $L=f^{*} L^{\prime}$ for some line bundle $L^{\prime}$ on $X^{\prime}$. Note that $L^{\prime}$ is nef because $L$ is nef and $f$ is surjective. Consequently $(X, L)$ and $\left(X^{\prime}, L^{\prime}\right)$ are birationally equivalent. In this case we go back to the starting point, apply the same argument as above to $\left(X^{\prime}, L^{\prime}\right)$ and continue in this manner.

This procedure must come to an end after a finite number of repetitions, since the Picard number $\rho(X)$ is finite. Therefore starting with an arbitrary semipolarized surface $(X, L)$, we end up with a semipolarized smooth surface $(Y, M)$ which is birationally equivalent to $(X, L)$ and satisfies one of the six conditions in the Theorem 1 . This completes the proof.

\section{§3. Proof of the Theorem 2}

Let $L$ be a nef line bundle on a projective normal surface $X$. The sectronal genus $g(X, L)$ of the semipolarized normal surface $(X, L)$ is given by the formula $2 g(X, L)-2=\left(\omega_{X}+L\right) L$, where $\omega_{X}$ is the canonical sheaf of $X$. 
We begin with the following

DEFINITION. Let $(X, L)$ be a semipolarized surface. A semipolarized smooth surface $(Y, M)$ is called an adjointly minimal model of $(X, L)$ if $(Y, M)$ is birationally equivalent to $(X, L)$ and satisfies one of the six conditions in the Theorem 1.

To prove the Theorem 2, we need the following two lemmas.

LEMMA 3.1. Let $f: X \rightarrow Y$ be a birational morphism of projective normal surfaces. Then $g(Y, M)=g(X, f * M)$ for any line bundle $M$ on $Y$.

For the proof, we refer to $[F]$, Lemma 1.8 .

Lemma 3.2. Let $Y$ be a $\boldsymbol{P}^{1}$-bundle over a smooth curve $C$ of genus $g$ and $M$ a nef line bundle on $Y$.

(3.2.1) If $(Y, M)$ is a scroll over $C$, then $g(Y, M)=g$.

(3.2.2) If $M$ is the pull-back of a line bundle $B$ of non-negative degree on $C$, then $g(Y, M)=1-\operatorname{deg} B$.

Proof. With the same notation as in (1.3), we have

$$
K_{Y} \equiv-2 C_{0}+(2 g-2-e) F
$$

For any nef line bundle $M$ on $Y$, we can write $M=a H+\pi^{*} B$ for some line bundle $B$ on $C$. Set $b=\operatorname{deg} B$. Then

$$
M \equiv a C_{0}+b F
$$

and an easy calculation shows that

$$
2 g(Y, M)-2=\left(K_{Y}+M\right) M=2 a(g-1)+(a-1)(2 b-a e) .
$$

(3.2.1) If $a=1$, then $g(Y, M)=g$.

(3.2.2) If $a=0$, then $g(Y, M)=1-b$, and we are done.

Proof of the Theorem 2. Let $(X, L)$ be a semipolarized normal surface. First of all, by virtue of Lemma 3.1 it suffices to consider an adjointly minimal model $(Y, M)$ of $(X, L)$.

Assume that

Then

$$
g(X, L)=g(Y, M) \leqq 0 .
$$

$$
\left(K_{Y}+M\right) M=2 g(Y, M)-2 \leqq-2,
$$

so that $K_{Y}+M$ is not nef. In case (2) of the Theorem 1,

$$
g(Y, M)=g\left(\boldsymbol{P}^{2}, \boldsymbol{O}_{\boldsymbol{P}}\right)=1,
$$


a contradiction. In case (3) or (4) of the Theorem 1 we see that

$$
g(Y, M)=0 .
$$

In case (5), if we let $(Y, M)$ be a scroll over a smooth curve $C$ of genus $g$, then

$$
0 \leqq g=g(Y, M) \leqq 0
$$

by (3.2.1). Consequently $C \cong \boldsymbol{P}^{1}$ and $g(Y, M)=0$. In case (6) we can write

$$
M \cong \pi * B
$$

for some line bundle $B$ of non-negative degree on $C$, where $\pi$ is the projection $Y \rightarrow C$. Then (3.2.2) tells us that

$$
1-\operatorname{deg} B=g(Y, M) \leqq 0,
$$

so $\operatorname{deg} B \geqq 1$.

Thus, in sum, $g(X, L)=g(Y, M) \geqq 0$ unless $Y$ is a $\boldsymbol{P}^{1}$-bundle over a smooth curve $C$ and $M \cong \pi * B$ for some line bundle $B$ on $C$ with $\operatorname{deg} B \geqq 2$. Moreover, if $g(X, L)=0$, then $(Y, M)$ is one of the following.

(1) $(Y, M) \cong\left(\boldsymbol{P}^{2}, \boldsymbol{O}_{\boldsymbol{P}}(1)\right)$.

(2) $(Y, M) \cong\left(\boldsymbol{P}^{2}, \mathcal{O}_{\boldsymbol{P}}(2)\right)$.

(3) $(Y, M)$ is a scroll over $\boldsymbol{P}^{\mathbf{1}}$.

(4) $Y$ is a $\boldsymbol{P}^{1}$-bundle over a smooth curve $C$ and $M \cong \pi^{*} B$ for some line bundle $B$ on $C$ with $\operatorname{deg} B=1$.

This completes the proof.

\section{$\S 4$. Proof of the Theorem 3}

Let $(X, L)$ be a semipolarized normal surface of sectional genus one. By Lemma 3.1 it suffices to consider an adjointly minimal model $(Y, M)$ of $(X, L)$. Since $g(Y, M)=g(X, L)=1$, we have

$$
\left(K_{Y}+M\right) M=2 g(Y, M)-2=0 .
$$

First of all, if $M$ is numerically trivial, then $g(Y, M)=1$; thus we may assume from the start that $M$ is not numerically trivial.

(4.1) Clearly $(Y, M)$ is neither $\left(\boldsymbol{P}^{2}, \mathcal{O}_{\boldsymbol{P}}\right)$, nor $\left(\boldsymbol{P}^{2}, \mathcal{O}_{\boldsymbol{P}}(1)\right)$, nor $\left(\boldsymbol{P}^{2}, \mathcal{O}_{\boldsymbol{P}}(2)\right)$. Thus, if $K_{Y}+M$ is not nef, then from Theorem 1 one of the following holds.

(1) $(Y, M)$ is a scroll over a smooth curve $C$.

(2) $Y$ is a $\boldsymbol{P}^{1}$-bundle over a smooth curve $C$ and $M$ is the pull-back of a line bundle $B$ of non-negative degree on $C$.

In case (1) we see that $C$ is an elliptic curve since the genus of $C$ is $g(Y, M)$ by virtue of Lemma 3.2. Assume that case (2) holds. Then it follows from Lemma 3.2 that $1-\operatorname{deg} B=g(Y, M)$, and hence that $\operatorname{deg} B=0$. Thus $M$ 
is numerically trivial, contrary to assumption.

So, from now on, we assume that $K_{Y}+M$ is nef.

(4.2) Since $M$ is nef, we have $M^{2} \geqq 0$. Assume that $M^{2}>0$. Then $M$ is big. Since $\left(K_{Y}+M\right) M=0$, we use the Hodge index theorem to see that

$$
K_{Y}+M \equiv 0,
$$

and hence $Y$ is a weak Del Pezzo surface. Thus the numerical equivalence implies the linear equivalence, and so $M=-K_{Y}$.

From now on, we suppose that $M^{2}=0$, so that $K_{Y} M=0$. Note that since $K_{Y}+M$ is nef,

$$
0 \leqq\left(K_{Y}+M\right)^{2}=K_{Y}^{2} .
$$

We claim that $K_{Y}^{2}=0$. To see this, suppose that $K_{Y}^{2}>0$. Then we have $M \equiv 0$ by the Hodge index theorem since $K_{Y} M=0$, a contradiction. Thus $K_{Y}^{2}=0$. We shall have to use two different approaches, depending on whether $K_{Y}$ is nef or not.

(4.3) Suppose first that $K_{Y}$ is nef. Then, unless $K_{Y} \equiv 0, Y$ is a minimal surface with Kodaira dimension 1.

(4.4) From now on, we suppose that $K_{Y}$ is not nef. Then Theorem 1.1 tells us that $Y$ has an extremal ray $R$ which is spanned by an extremal rational curve $l$. By Theorem 1.2, since $K_{Y}^{2}=0$, one of the following holds.

(i) $Y$ is $a P^{1}$-bundle over a smooth curve $C$.

(ii) There exists at least one (-1)-curve.

(4.5) In case (i), since $K_{Y}^{2}=0, C$ must be an elliptic curve. With the same notation as in (1.3), we have $K_{Y} \equiv-2 C_{0}-e F$. Now write $M \equiv a C_{0}+b F$. Then

$$
K_{Y}+M \equiv(a-2) C_{0}+(b-e) F .
$$

Since $K_{Y}+M$ is nef,

$$
0 \leqq\left(K_{Y}+M\right) F=a-2,
$$

i. e., $a \geqq 2$. Moreover,

$$
0=M^{2}=a(2 b-a e),
$$

and so $2 b-a e=0$. Now we know $e \geqq-1$. If $e \geqq 0$, then we have by Lemma 1.4

$$
b \geqq a e=2 b \geqq 0,
$$

so that $b=e=0$. On the other hand, if $e=-1$, then $b=-a / 2$ and $\mathcal{E}$ is indecomposable. Conversely, if $e=0$ (resp. $=-1$ ) and $a \geqq 2$, we use Lemma 1.4 (resp. Lemma 1.5) to see that $M \equiv a C_{0}$ (resp. $\left.\equiv a C_{0}-(a / 2) F\right)$ is nef.

(4.6) Assume that case (ii) holds. The argument now splits up into two 
cases, depending on whether $2 K_{Y}+M$ is nef or not.

(4.7) If $2 K_{Y}+M$ is not nef, then by the cone Theorem 1.1 there exists an extremal rational curve $E$ such that $\left(2 K_{Y}+M\right) E<0$. Since $Y$ is not a $\boldsymbol{P}^{1}$-bundle over a smooth curve, Theorem 1.2 tells us that $E$ must be a (-1)-curve; thus $M E<2$. On the other hand, since $K_{Y}+M$ is nef, we have

$$
\left(K_{Y}+M\right) E \geqq 0,
$$

i. e., $M E \geqq 1$. Thus $M E=1$.

Let $f: Y \rightarrow Z$ be the blow-down of $E$. Then $M+\mathcal{O}_{Y}(E)=f * N$ for some line bundle $N$ on $Z$ because $\left(M+\mathcal{O}_{Y}(E)\right) E=0$. Let $C$ be an integral curve on $Z, r$ the multiplicity of $C$ at the point $f(E)$, and $\widetilde{C}$ the proper transform of $C$ in $Y$. Then

so

$$
\tilde{C}=f^{*} C-r E,
$$

$$
\begin{aligned}
M \tilde{C} & =\left(f^{*} N-\mathcal{O}_{Y}(E)\right)\left(f^{*} C-r E\right) \\
& =N C-r .
\end{aligned}
$$

Since $M$ is nef, we deduce that

$$
N C \geqq r \geqq 0 .
$$

Thus $N$ is nef. Moreover,

$$
N^{2}=\left(M+\mathcal{O}_{Y}(E)\right)^{2}=1,
$$

and so $N$ is big. Note that since $K_{Y}=f * K_{Z}+\mathcal{O}_{Y}(E)$,

$$
K_{Y}+M=f^{*}\left(K_{Z}+N\right) \text {. }
$$

Consequently $K_{Z}+N$ is nef and we obtain

$$
\begin{aligned}
0 & =\left(K_{Y}+M\right) M \\
& =f^{*}\left(K_{Z}+N\right)\left(f^{*} N-\mathcal{O}_{Y}(E)\right) \\
& =\left(K_{Z}+N\right) N
\end{aligned}
$$

so that by the Hodge index theorem,

$$
N=-K_{Z},
$$

i. e., $Z$ is a weak Del Pezzo surface with $\left(-K_{Z}\right)^{2}=1$. Thus

$$
\begin{aligned}
M & =f^{*} N-\mathcal{O}_{Y}(E) \\
& =f^{*}\left(-K_{Z}\right)-\mathcal{O}_{Y}(E)=-K_{Y} .
\end{aligned}
$$

(4.8) Assume that $2 K_{Y}+M$ is nef. Let 


$$
c=\min \left\{t \in N \mid t K_{Y}+M \text { is not nef }\right\} .
$$

Then $c \geqq 3$. Set $A=(c-2) K_{Y}+M$. Then $A$ is nef and we have

$$
\left(K_{Y}+A\right) A=\left((c-1) K_{Y}+M\right)\left((c-2) K_{Y}+M\right)=0,
$$

and of course

$$
K_{Y}^{2}=K_{Y} A=A^{2}=0 .
$$

Since $K_{Y}+A$ is nef and $2 K_{Y}+A$ is not nef, we deduce that the semipolarized smooth surface $(Y, A)$ satisfies the condition (4.7). Thus $A=-K_{Y}$, i. e., $M=$ $-(c-1) K_{Y}$ with $c-1 \geqq 2$. Combining this with (4.7), we conclude that in case (ii), $Y$ is the blow-up of $Z$ at a point, where $Z$ is a weak Del Pezzo surface with $\left(-K_{Z}\right)^{2}=1$. Moreover, we have $M=-a K_{Y}$ with $a \geqq 1$. This completes the proof.

Remark. Finally, we give three examples of type (1), (2) and (6) in the Theorem 3 respectively.

(1) Let $Y$ be a minimal elliptic surface with Kodaira dimension 1 and $p: Y \rightarrow B$ the elliptic fibration. Let $M$ be the line bundle associated to a smooth elliptic fiber of $p$. Then $K_{Y} M=M^{2}=0$ and $M$ is nef.

(2) Let $Y$ be the product of two elliptic curves $C$ and $D$. Then $K_{Y}=\mathcal{O}_{Y}$. Let $M$ be the line bundle associated to a fiber of $Y \rightarrow C$. Then $M^{2}=0$ and $M$ is nef.

(6) Let $Z$ be a weak Del Pezzo surface with $\left(-K_{Z}\right)^{2}=1$. Then it follows from [D], p. 39, Théorème 1 that $\left|-K_{Z}\right|$ contains a smooth irreducible curve $C$. Take an arbitrary point $p$ of $C$. Let $f: Y \rightarrow Z$ be the blow-up of $Z$ at $p$, $E=f^{-1}(p)$ the $(-1)$-curve of the blow-up, and $\tilde{C}$ the proper transform of $C$ in $Y$. Then

$$
\widetilde{C}=f^{*} C-E \in\left|f^{*}\left(-K_{Z}\right)-\mathcal{O}_{Y}(E)\right|=\left|-K_{Y}\right|,
$$

i. e., $-K_{Y}$ is represented by the irreducible curve $\tilde{C}$ with $\tilde{C}^{2}=0$. Thus $-K_{Y}$ is nef and consequently $M=-a K_{Y}$ is nef for any $a \geqq 1$.

\section{REFERENCES}

[D] M. Demazure, Surfaces de Del Pezzo, Séminare sur les Singularités des Surfaces (M. Demazure, H. Pinkham, B. Teissier, eds.), Lecture Notes in Math., 777, Springer-Verlag, Berlin-Heidelberg-New York, 1980, pp. 21-69.

[F] T. FujitA, Remarks on quasi-polarized varieties, Nagoya Math. J., 115 (1989), 105-123.

[H] R. HARtshorne, Algebraic Geometry, Graduate Texts in Math., 52, SpringerVerlag, New York-Heidelberg-Berlin, 1977.

[LP] A. LANTERI AND M. PAlleschi, About the adjunction process for polarized algebraic surfaces, J. Reine Angew. Math., 352 (1984), 15-23. 
[Ma] H. MAedA, Surfaces with nef anticanonical bundles, Boll. Un. Mat. Ital. A(7), 8 (1994), 425-430.

[Mo] S. MoRI, Threefolds whose canonical bundles are not numerically effective, Ann. of Math., 116 (1982), 133-176.

Graduate School of Mathematics

KYUSHU UNIVERSITY

4-2-1 Ropponmatsu, ChuO-KU

FUKUOKA 810

JAPAN

e-mail : hmaeda@math.kyushu-u.ac.jp 
